Document downloaded from:

http://hdl.handle.net/10251/155435

This paper must be cited as:

Bonet Solves, JA.; Lusky, W.; Taskinen, J. (2019). Distance formulas on weighted Banach spaces of analytic functions. Complex Analysis and Operator Theory. 13(3):893-900. https://doi.org/10.1007/s11785-018-0815-4

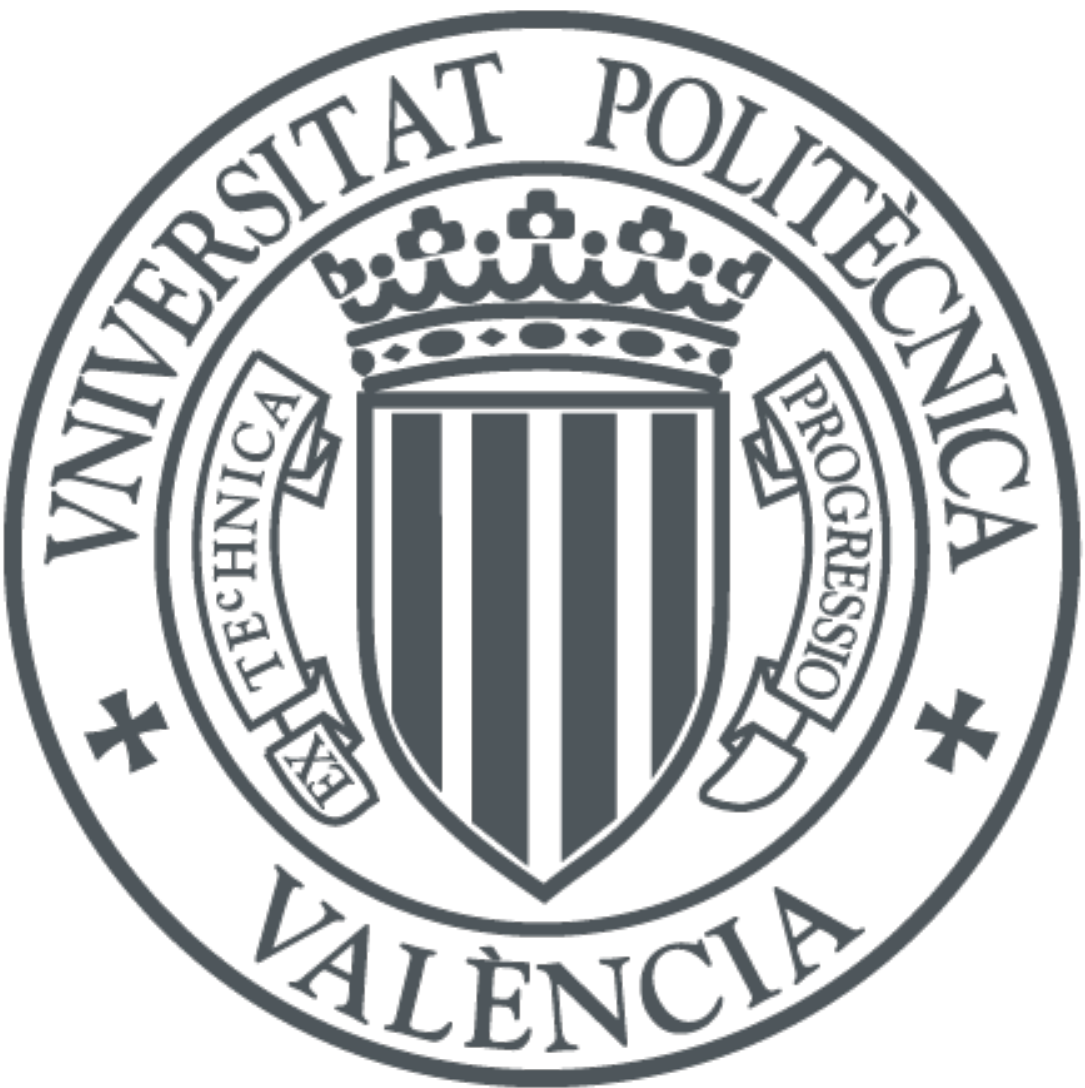

The final publication is available at

https://doi.org/10.1007/s11785-018-0815-4

Copyright Springer-Verlag

Additional Information 


\title{
DISTANCE FORMULAS ON WEIGHTED BANACH SPACES OF ANALYTIC FUNCTIONS
}

\author{
JOSÉ BONET, WOLFGANG LUSKY, AND JARI TASKINEN
}

\begin{abstract}
Let $v$ be a radial weight function on the unit disc or on the complex plane. It is shown that for each analytic function $f_{0}$ in the Banach space $H_{v}^{\infty}$ of all analytic functions $f$ such that $v|f|$ is bounded, the distance of $f_{0}$ to the subspace $H_{v}^{0}$ of $H_{v}^{\infty}$ of all the functions $g$ such that $v|g|$ vanishes at infinity is attained at a function $g_{0} \in H_{v}^{0}$. Moreover a simple, direct proof of the formula of the distance of $f$ to $H_{v}^{0}$ due to Perfekt is presented. As a consequence the corresponding results for weighted Bloch spaces are obtained.
\end{abstract}

\section{INTRODUCTION AND NOTATION.}

Let us introduce some notation and terminology. We set $R=1$ (for the case of holomorphic functions on the unit disc) and $R=+\infty$ (for the case of entire functions). A weight $v$ is a continuous function $v:[0, R[\rightarrow] 0, \infty[$, which is nonincreasing on $\left[0, R\right.$ [ and satisfies $\lim _{r \rightarrow R} r^{n} v(r)=0$ for each $n \in \mathbb{N}$. We extend $v$ to $\mathbb{D}$ if $R=1$ and to $\mathbb{C}$ if $R=+\infty$ by $v(z):=v(|z|)$. For such a weight $v$, we define the Banach space $H_{v}^{\infty}$ of analytic functions $f$ on the disc $\mathbb{D}$ (if $R=1$ ) or on the whole complex plane $\mathbb{C}$ (if $R=+\infty$ ) such that $\|f\|_{v}:=\sup _{|z|<R} v(z)|f(z)|<\infty$. For an analytic function $f \in H(\{z \in \mathbb{C} ;|z|<R\})$ and $r<R$, we denote $M(f, r):=$ $\max \{|f(z)| ;|z|=r\}$. Using the notation $O$ and $o$ of Landau, $f \in H_{v}^{\infty}$ if and only if $M(f, r)=O(1 / v(r)), r \rightarrow R$.

It is known that the closure of the polynomials in $H_{v}^{\infty}$ coincides with the Banach space $H_{v}^{0}$ of all those analytic functions on $\{z \in \mathbb{C} ;|z|<R\}$ such that $M(f, r)=$ $o(1 / v(r)), r \rightarrow R$. see e.g. [2].

Spaces of type $H_{v}^{\infty}$ appear in the study of growth conditions of analytic functions and have been investigated in various articles since the work of Shields and Williams, see e.g. [2],[3], [5], [6], [9] and the references therein.

We recall some examples of weights:

For $R=1$,

(i) $v(r)=(1-r)^{\alpha}$ with $\alpha>0$, which are the standard weights on the disc,

(ii) $v(r)=\exp \left(-(1-r)^{-1}\right)$, and

(iii) $v(z)=\left(\log \frac{e}{1-r}\right)^{-\alpha}, \alpha>0$

For $R=+\infty$,

(i) $v(r)=\exp \left(-r^{p}\right)$ with $p>0$

(ii) $v(r)=\exp (-\exp r)$, and

(iii) $v(r)=\exp \left(-\left(\log ^{+} r\right)^{p}\right)$, where $p \geq 2$ and $\log ^{+} r=\max (\log r, 0)$.

Given an analytic function $f$ on $\mathbb{D}$ or $\mathbb{C}$, we denote by $\sigma_{n} f$ the $n$ 'th Cesaro mean of $f$; i.e. the arithmetic mean of the first $n$ Taylor polynomials of $f$. In this case, one has $M\left(\sigma_{n} f, r\right) \leq M(f, r)$ for each $0<r<R$.

Key words and phrases. Banach spaces of analytic functions, weight, distance, Bloch functions. Mathematics Subject Classification 2010: Primary 46E15; Secondary 30D45. 
In this note we investigate the distance $d\left(f, H_{v}^{0}\right)=\inf _{g \in H_{v}^{0}}\|f-g\|_{v}$ of a function $f \in H_{v}^{\infty}$ to the closed subspace $H_{v}^{0}$. Perfekt in Example 4.4 of [7] proved that $d\left(f, H_{v}^{0}\right)=\limsup _{r \rightarrow R} M(f, r) v(r)$ for each $f \in H_{v}^{\infty}$. This result follows from an abstract result [7, Theorem 2.3] with an argument using duality and measures. It implies Theorem 3.9 and Corollary 6.4 in Tjani [10] about the distance of a Bloch function to the little Bloch space. The result of Tjani only gives an estimate, not equality. There are some other recent papers dealing with distance formulas. See [11] and the references therein.

Our main result is Theorem 2.2. It shows that $H_{v}^{0}$ is a proximinal subspace of $H_{v}^{\infty}$; that is, it proves that for each $f \in H_{v}^{\infty}$ the distance $d\left(f, H_{v}^{0}\right)$ is attained at a point $g \in H_{v}^{0}$. Moreover, it gives an elementary, direct, but not trivial, proof of the formula of the distance due to Perfekt [7]. The corresponding result for the case of Bloch type functions is obtained as a consequence in Corollary 2.5.

\section{Results.}

Given $f \in H_{v}^{\infty}$ we clearly have

$$
\limsup _{|z| \rightarrow R} v(z)|f(z)|=\limsup _{r \rightarrow R} M(f, r) v(r)=\lim _{r \rightarrow R} \sup _{s \geq r} v(s) M(f, s) .
$$

Remark 2.1. It is easy to see that, for each $f \in H_{v}^{\infty}$,

$$
\limsup _{r \rightarrow R} M(f, r) v(r)=\inf _{g \in H_{v}^{0}} \limsup _{r \rightarrow R} M(f-g, r) v(r)
$$

Indeed, this follows from the fact that

$$
\limsup _{r \rightarrow R} M(g, r) v(r)=0 \quad \text { for every } \quad g \in H_{v}^{0} .
$$

Theorem 2.2. For every $f \in H_{v}^{\infty}$ there is $g \in H_{v}^{0}$ with

$$
d\left(f, H_{v}^{0}\right)=\|f-g\|_{v}=\limsup _{r \rightarrow R} M(f, r) v(r) .
$$

To prove the theorem we begin with the following

Lemma 2.3. Let $f \in H_{v}^{\infty}$ and assume that there is $\tau<1$ with

$$
\tau\|f\|_{v} \leq \limsup _{r \rightarrow R} M(f, r) v(r) .
$$

Then, for each $\varepsilon>0$ and $m \in \mathbb{N}$ there is $n \in \mathbb{N}, n>m$, such that with $\rho=$ $(1-\tau) /(1+\tau)$ we have

$$
\left(\frac{1+\tau}{2(1+\varepsilon)}\right)\left\|f-\rho \sigma_{n} f\right\|_{v} \leq \limsup _{r \rightarrow R} M(f, r) v(r)=\limsup _{r \rightarrow R} M\left(f-\rho \sigma_{n} f, r\right) v(r) .
$$

Proof. The last equality follows from the facts that $\sigma_{n} f \in H_{v}^{0}$ and that for each element $g \in H_{v}^{0}$ we have $\lim _{\sup _{r \rightarrow R}} M(g, r) v(r)=0$.

Fix $\varepsilon>0$ and $m \in \mathbb{N}$. By the definition of limsup there is $r_{0}<R$ such that

$$
\begin{aligned}
\sup _{r_{0} \leq r<R} M(f, r) v(r) & \leq(1+\varepsilon) \inf _{0<s<R} \sup _{s \leq r<R} M(f, r) v(r) \\
& =(1+\varepsilon) \limsup _{r \rightarrow R} M(f, r) v(r) .
\end{aligned}
$$


Since $f$ is continuous on $r_{0} \overline{\mathbb{D}}$, the $n$ 'th Cesaro means of $f$ satisfy $\sigma_{n} f \rightarrow f$ as $n \rightarrow \infty$ uniformly on $r_{0} \overline{\mathbb{D}}$. Put

and fix $0<\delta$ such that

$$
\rho:=\frac{1-\tau}{1+\tau}
$$

$$
\left(\delta+\frac{2 \tau}{1+\tau}\right) \leq(1+\varepsilon) \frac{2 \tau}{1+\tau} .
$$

For $0 \leq r \leq r_{0}$ we obtain $M\left(f-\sigma_{n} f, r\right) v(r)<\delta\|f\|_{v}$ if $n>m$ is large enough. Hence

$$
\begin{aligned}
M\left(f-\rho \sigma_{n} f, r\right) v(r) & \leq(1-\rho) M(f, r) v(r)+\rho M\left(f-\sigma_{n} f, r\right) v(r) \\
& \leq((1-\rho)+\delta)\|f\|_{v} .
\end{aligned}
$$

If $r_{0} \leq s<R$ then we have, in view of (1),

$$
M\left(f-\rho \sigma_{n} f, s\right) v(s) \leq(1+\rho) M(f, s) v(s) \leq(1+\varepsilon)(1+\rho) \limsup _{r \rightarrow R} M(f, r) v(r)
$$

From the definition of $\rho$ we get

$$
(1+\varepsilon)(1+\rho)=\frac{2(1+\varepsilon)}{1+\tau}
$$

and

$$
1-\rho=\frac{2 \tau}{1+\tau}
$$

Hence (1), (2), (3), (4) and the assumption of the lemma yield

$$
\begin{aligned}
\left\|f-\rho \sigma_{n} f\right\|_{v} & =\sup _{0 \leq r<R} M\left(f-\rho \sigma_{n} f, r\right) v(r) \\
& \leq \max \left((\delta+(1-\rho))\|f\|_{v},(1+\varepsilon)(1+\rho) \limsup _{r \rightarrow R} M(f, r) v(r)\right) \\
& \leq \max \left(\left(\delta+\frac{2 \tau}{1+\tau}\right)\|f\|_{v},\left(\frac{2(1+\varepsilon)}{1+\tau}\right) \limsup _{r \rightarrow R} M(f, r) v(r)\right) \\
& \leq\left(\frac{2(1+\varepsilon)}{1+\tau}\right) \limsup _{r \rightarrow R} M(f, r) v(r) .
\end{aligned}
$$

The proof is complete.

Proof. (of Theorem 2.2) Let $f \in H_{v}^{\infty}$. If $\limsup _{r \rightarrow R} M(f, r) v(r)=0$ then $f \in H_{v}^{0}$ and $d\left(f, H_{v}^{0}\right)=0$.

Now assume that $\limsup _{r \rightarrow R} M(f, r) v(r)>0$ and find $\tau_{0}<1$ with

$$
\|f\|_{v} \leq \frac{1}{\tau_{0}} \limsup _{r \rightarrow R} M(f, r) v(r) .
$$

Put $\rho_{1}=\left(1-\tau_{0}\right) /\left(1+\tau_{0}\right)$ and $f_{0}=f$.

We proceed by induction and suppose that we have already selected $\tau_{0}<\tau_{m-1}<$ $\tau_{m}<1, \rho_{m}>0$ and $f_{m}:=f-\sum_{k=1}^{m} \rho_{k} \sigma_{n_{k}} f_{k-1}$ for some $n_{m}>n_{m-1}$ with $\left\|f_{m}\right\|_{v}<$ $\left(1 / \tau_{m}\right) \lim \sup _{r \rightarrow R} M\left(f_{m}, r\right) v(r)$.

A simple calculation shows

$$
\frac{1-\tau_{m}}{3+\tau_{m}}<\left(\frac{2}{3}\right) \frac{1-\tau_{m}}{1+\tau_{m}} .
$$


Find $\varepsilon_{m}>0$ such that

$$
\varepsilon_{m}<\frac{1}{m}, \quad \frac{1+\tau_{m}}{2\left(1+\varepsilon_{m}\right)}>\tau_{m}
$$

and

$$
\frac{1-\frac{1+\tau_{m}}{2\left(1+\varepsilon_{m}\right)}}{1+\frac{1+\tau_{m}}{2\left(1+\varepsilon_{m}\right)}}=\frac{1+2 \varepsilon_{m}-\tau_{m}}{3+2 \varepsilon_{m}+\tau_{m}}<\left(\frac{2}{3}\right) \frac{1-\tau_{m}}{1+\tau_{m}} .
$$

Put

$$
\tau_{m+1}:=\frac{1+\tau_{m}}{2\left(1+\varepsilon_{m}\right)} \quad \text { and } \quad \rho_{m+1}:=\frac{1-\tau_{m}}{1+\tau_{m}}=\frac{1+2 \varepsilon_{m}-\tau_{m-1}}{3+2 \varepsilon_{m}+\tau_{m-1}} .
$$

Observe that $\tau_{m}<\tau_{m+1}<1$. Then Lemma 2.3 yields $n_{m+1}>n_{m}$ such that, with

$$
f_{m+1}:=f_{m}-\rho_{m+1} \sigma_{n_{m+1}} f_{m}=f-\sum_{k=1}^{m+1} \rho_{k} \sigma_{n_{k}} f_{k-1},
$$

we have

$$
\begin{array}{r}
\left\|f_{m+1}\right\|_{v} \leq \frac{1}{\tau_{m+1}} \limsup _{r \rightarrow R} M\left(f_{m+1}, r\right) v(r) \\
\quad=\frac{1}{\tau_{m+1}} \limsup _{r \rightarrow R} M(f, r) v(r) .
\end{array}
$$

(5) and (7) yield $\lim _{m \rightarrow \infty} \tau_{m}=1$ since $\left(\tau_{m}\right)$ is an increasing bounded sequence. On account of (6) we obtain

$$
\rho_{m+1} \leq\left(\frac{2}{3}\right) \rho_{m} \quad \text { for all } m
$$

hence,

$$
\rho_{m} \leq\left(\frac{2}{3}\right)^{m} \rho_{0}
$$

This implies that $\sum_{k=1}^{\infty} \rho_{k} \sigma_{n_{k}} f_{k-1}$ converges to an element $g \in H_{v}^{0}$, since $\left\|\sigma_{n_{k}} f_{k-1}\right\|_{v} \leq$ $\left\|f_{k-1}\right\|_{v} \leq \tau_{k-1}^{-1}\|f\|_{v} \leq \tau_{0}^{-1}\|f\|_{v}$ for all $k$, as it follows from (9). Therefore, we can apply (8) and (9) to get

$$
\begin{gathered}
\quad\|f-g\|_{v} \leq\left\|f_{m+1}\right\|_{v}+\left\|\sum_{k=m+2}^{\infty} \rho_{k} \sigma_{n_{k}} f_{k-1}\right\|_{v} \leq \\
\leq \frac{1}{\tau_{m+1}} \limsup _{r \rightarrow R} M(f, r) v(r)+\rho_{0} \tau_{0}^{-1} \sum_{k=m+2}^{\infty}\left(\frac{2}{3}\right)^{k} .
\end{gathered}
$$

Thus

$$
\|f-g\|_{v} \leq \limsup _{r \rightarrow R} M(f, r) v(r)=\inf _{h \in H_{v}^{0}} \limsup _{r \rightarrow R} M(f-h, r) v(r) \leq d\left(f, H_{v}^{0}\right) .
$$

We conclude $d\left(f, H_{v}^{0}\right)=\|f-g\|_{v}=\limsup _{r \rightarrow R} M(f, r) v(r)$.

One of the referees pointed out that our construction reminded her/him of a construction in [1], where the authors prove a proximinality result for bounded operators. 
Remark 2.4. The following simple examples show that the distance $d\left(f, H_{v}^{0}\right)$ can be attained at many points of $H_{v}^{0}$ for a given function $f \in H_{v}^{\infty}$.

(1) Consider the weight $v(r)=e^{-r}, r \in[0, \infty[$, on the complex plane and the analytic function $f(z)=e^{z}, z \in \mathbb{C}$. Clearly $f \in H_{v}^{\infty}$ and $\|f\|_{v}=1$. Set $P_{n}(z)=$ $\sum_{k=0}^{n} \frac{z^{k}}{k !}$ for each $n \in \mathbb{N}$. We have, for each $n, P_{n} \in H_{v}^{0}$ and

$$
\left\|f-P_{n}\right\|_{v}=\sup _{r>0} e^{-r} \sum_{k=n+1}^{\infty} \frac{r^{k}}{k !}=1=d\left(f, H_{v}^{0}\right) .
$$

(2) Now define the weight $v(r)=1-r, r \in[0,1[$, on the unit disc. The function $f(z)=\frac{1}{1-z}=\sum_{k=0}^{\infty} z^{k}$ belongs to $H_{v}^{\infty}$ and $\|f\|_{v}=1$. Set $P_{n}(z)=\sum_{k=0}^{n} z^{k}$ for each $n \in \mathbb{N}$. We have, for each $n, P_{n} \in H_{v}^{0}$ and

$$
M\left(f-P_{n}, r\right)=\sum_{k=n+1}^{\infty} r^{k}=\frac{r^{n+1}}{1-r} .
$$

Therefore

$$
\left\|f-P_{n}\right\|_{v}=\sup _{r \in[0,1[}(1-r) M\left(f-P_{n}, r\right)=1=d\left(f, H_{v}^{0}\right) .
$$

(3) The proximinality in Theorem 2.2, i.e. the existence of the minimizer $g$, also appears in Perfekt [8] as an abstract consequence of the fact that $H_{v}^{0}$ is an $M$-ideal of $H_{v}^{\infty}$. Moreover, by further abstract $M$-ideal theory, the minimizer for a given $f \in H_{v}^{\infty} \backslash H_{v}^{0}$ is never unique; see [4]. This was pointed out to us by one of the referees, who also emphasized that we give a very explicit construction, which these references do not.

Let $v$ be a weight on the unit disc $\mathbb{D}$; i.e. $R=1$. The weighted Bloch space is defined by

$$
\mathcal{B}_{v}=\left\{f \in H(\mathbb{D}): f(0)=0,\|f\|_{\mathcal{B}_{v}}=\sup _{z \in \mathbb{D}} v(z)\left|f^{\prime}(z)\right|<\infty\right\}
$$

and the little Bloch space

$$
\mathcal{B}_{v, 0}=\left\{f \in \mathcal{B}: \lim _{|z| \rightarrow 1} v(z)\left|f^{\prime}(z)\right|=0\right\} .
$$

They are Banach spaces endowed with the norm $\|\cdot\|_{\mathcal{B}_{v}}$.

The classical Bloch space $\mathcal{B}$ and little Bloch space $\mathcal{B}_{0}$ correspond to the weight $v(z):=1-|z|^{2}$. Among the many references on these spaces, we mention Zhu [12], for example.

Define the bounded operators $S: \mathcal{B}_{v} \rightarrow H_{v}^{\infty}, S(h)=h^{\prime}$ and $S^{-1}: H_{v}^{\infty} \rightarrow$ $\mathcal{B}_{v},\left(S^{-1} h\right)(z)=\int_{0}^{z} h(\xi) d \xi$. Then $S S^{-1}=i d_{H_{v}^{\infty}}, S^{-1} S=i d_{\mathcal{B}_{v}}$ and $S, S^{-1}$ are isometric onto maps. These operators induce isometries between $H_{v}^{0}$ and $\mathcal{B}_{v, 0}$.

The following result is a direct consequence of Theorem 2.2. It should be compared with Example 4.1 in [7]. It improves [10, Corollary 6.4].

Corollary 2.5. For each $f \in \mathcal{B}_{v}$ there is $g \in \mathcal{B}_{v, 0}$ such that

$$
d\left(f, \mathcal{B}_{v, 0}\right)=\|f-g\|_{\mathcal{B}_{v}}=\limsup _{r \rightarrow 1} M\left(f^{\prime}, r\right) v(r) .
$$

Finally we mention the weighted spaces of harmonic functions for a given weight $v$ on $\{z \in \mathbb{C} ;|z|<R\}$. Let $h_{v}^{\infty}$ consist of all harmonic functions on $\{z \in \mathbb{C} ;|z|<R\}$ with $\|f\|_{v}=\sup _{|z|<R}|f(z)| v(z)<\infty$ and let $h_{v}^{0}$ be the closure of all trigonometric 
polynomials in $h_{v}^{\infty}$. Using the arguments of the proof of Theorem 2.2. word by word yields

Theorem 2.6. For every $f \in h_{v}^{\infty}$ there is $g \in h_{v}^{0}$ with

$$
d\left(f, H_{v}^{0}\right)=\|f-g\|_{v}=\limsup _{r \rightarrow R} M(f, r) v(r) .
$$

Acknowledgements. (1) The authors are very thankful to the referees for their careful reading of the manuscript and their suggestions.

(2) The research of Bonet was partially supported by the projects MTM201676647-P and GV Prometeo 2017/102. The research of Taskinen was partially supported by the research grant from the Faculty of Science of the University of Helsinki.

\section{REFERENCES}

[1] S. Axler, I.D. Berg, N. Jewell, A. Shields, Approximation by compact operators and the space $H^{\infty}+C$, Ann. of Math. 109 (1979), 601-612.

[2] K.D. Bierstedt, J. Bonet, A. Galbis, Weighted spaces of holomorphic functions on bounded domains, Michigan Math. J. 40 (1993), 271-297.

[3] K.D. Bierstedt, J. Bonet, J. Taskinen, Associated weights and spaces of holomorphic functions, Studia Math. 127 (1998), 137-168.

[4] R. Holmes, B. Scranton, J. Ward, Approximation from the space of compact operators and other M-ideals, Duke Math. J. 42 (1975), 259-269.

[5] W.Lusky, On the Fourier series of unbounded harmonic functions, J. of the Lond. Math. Soc. (2) 61, 568-580, (2000)

[6] W.Lusky, On the isomorphism classes of weighted spaces of harmonic and holomorphic functions, Studia Math. 175, 19-45, (2006)

[7] K.-M. Perfekt, Duality and distance formulas in spaces defined by means of oscillation, Ark. Mat. 51 (2013), 345-361.

[8] K.-M. Perfekt, On M-ideals and $o-O$ type spaces, Math. Scand. 121 (2017), no. 1, 151-160.

[9] A.L. Shields, D.L. Williams, Bounded projections, duality and multipliers in spaces of analytic functions, Trans. Amer. Math. Soc. 162 (1971), 287-302.

[10] M. Tjani, Distance of a Bloch function to the little Bloch space, Bull. Austral. Math. Soc. 74 (2006), 101-119.

[11] C. Yuan, C. Tong, Distance from Bloch-type functions to the analytic space $F(p, q, s)$, Abstract Appl. Anal. 2014, article ID 610237, 7 pages.

[12] K. Zhu, Operator Theory in Function Spaces. Second Edition, Amer. Math. Soc., 2007.

\section{Authors' addresses:}

José Bonet (corresponding author): Instituto Universitario de Matemática Pura y Aplicada IUMPA, Universitat Politècnica de València, E-46071 Valencia, Spain email: jbonet@mat.upv.es

Wolfgang Lusky: FB 17 Mathematik und Informatik, Universität Paderborn, D33098 Paderborn, Germany.

email: lusky@uni-paderborn.de

Jari Taskinen: Department of Mathematics and Statistics, P.O. Box 68, University of Helsinki, 00014 Helsinki, Finland.

email: jari.taskinen@helsinki.fi 